\title{
Martensitic Transformation in Co-Based Ferromagnetic Shape Memory Alloy
}

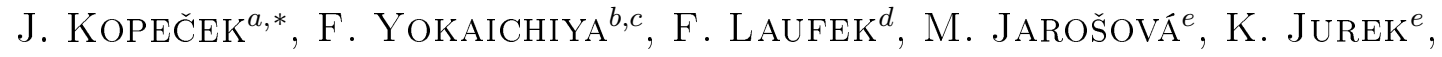
J. Drahokoupil ${ }^{a}$, S. Sedláková-Ignácováa ${ }^{a}, \mathrm{P}_{\text {. Molnár }}^{a}$ And O. HeCzko $^{a}$

${ }^{a}$ Institute of Physics of the AS CR, Na Slovance 2, 18221 Praha, Czech Republic

${ }^{b}$ Laboratório Nacional de Luz Síncrotron, Campinas, Brazil

${ }^{c}$ Helmholtz-Zentrum Berlin, Hahn-Meitner-Platz 1, 14109 Berlin, Germany

${ }^{d}$ Czech Geological Survey, Geologická 6, 15200 Praha 5, Czech Republic

${ }^{e}$ Institute of Physics of the AS CR, Cukrovarnická 10/112, 16200 Praha, Czech Republic

The $\mathrm{Co}_{38} \mathrm{Ni}_{33} \mathrm{Al}_{29}$ alloy in both powder and bulk state was investigated in the presented study using neutron diffraction on E9 high resolution powder diffractometer at HZB (BER II). The reverse martensitic phase transformation from the tetragonal martensitic phase into the cubic austenitic phase was observed with the phase coexistence within the temperatures from $183 \mathrm{~K}$ to $133 \mathrm{~K}$. The fcc cobalt solid solution particles distributed in the transforming matrix remained in the same state through the whole temperature range. The obtained results agree with magnetization measurements on the same annealed sample. The powder data are compared with experiments on bulk sample, although there was a texture present. The obtained results provide further information about the phase transition process in this important class of ferromagnetic shape memory alloys.

PACS: 78.40.Kc, 61.05.fm, 81.30.Kf, 81.40.Ef

\section{Introduction}

Cobalt-based shape memory alloys belong to the less understood ferromagnetic shape memory alloys (FSMAs) [1]. Although their magneto-elastic properties are not as promising as in $\mathrm{Ni}-\mathrm{Mn}-\mathrm{Ga}$ alloys [2], their mechanical properties and superelastic behavior in a wide temperature range are very interesting for possible applications. Cobalt based FSMAs have a complicated two-phase microstructure even at high temperature in the austenitic state [3]. The B2 ordered matrix $(\mathrm{Co}, \mathrm{Ni}) \mathrm{Al}$ (space group $P$ m3m) undergoes martensitic transformation into the $L 1_{0}$ tetragonal phase (space group $P 4 / \mathrm{mmm}$ ), while the disordered cobalt solid solution particles remain in the $A 1$, face centered cubic (fcc) phase (space group Fm3m) and help to accommodate stresses during transformations. The transformation mechanism is very similar to that in the $\mathrm{Ni}-\mathrm{Al}$ alloys [4].

Previous studies on the $\mathrm{Co}_{38} \mathrm{Ni}_{33} \mathrm{Al}_{29}$ alloy demonstrated strong dependence of its transformation properties on the thermomechanical history, mainly the crystallization velocity $[5,6]$ and the same behavior was noticed on similar alloys as well [7]. Since it is known that the transformation can be affected by the complex distribution of the fcc cobalt solid solution particles within the $B 2$ matrix in the bulk material, we performed neutron diffraction experiments both on powder material and on quickly solidified bulk polycrystals with homogeneous distribution of the fcc particles.

\footnotetext{
* corresponding author; e-mail: kopecek@fzu.cz
}

\section{Experimental details}

The samples were prepared from the re-melted material, which was cooled down in the resistance heated furnace under an argon atmosphere. The bulk material was grinded mechanically in the planetary mill. The powders were sealed in argon filled silica tubes and annealed for $1 \mathrm{~h}$ at $1350^{\circ} \mathrm{C}$, then cooled down slowly out of the furnace. The polycrystalline sample was taken from the same material and was annealed for $1 \mathrm{~h}$ at $1275^{\circ} \mathrm{C}$ in argon and then quenched into cold water.

Quantitative phase analysis was performed using neutron diffraction experiments on a high resolution powder diffractometer E9 at HZB (BER II) Berlin as a function of temperature during heating. Data were collected up to $158^{\circ} 2 \Theta$, the acquisition time of each diffractogram was $4 \mathrm{~h}$.

The neutron diffraction patterns were refined using the FullProf software. Concerning the occupancy parameters of the sublattices we suppose that nickel and cobalt occupy their positions randomly. If aluminum atoms are lacking in the aluminum sublattice the corresponding positions are occupied by anti-site random mixture of cobalt and nickel atoms.

\section{Results}

\subsection{Powder samples}

Neutron diffraction studies were performed at eight temperatures from $375 \mathrm{~K}$ down to $73 \mathrm{~K}$. The diffraction patterns showed that the sample contains matrix and particles of fcc-cobalt solid solution ( $A 1$ phase). The matrix consists of tetragonal $L 1_{0}$ martensitic phase at the lowest temperatures and through reverse martensitic transformation its structure changes to cubic $B 2$ structure at higher temperatures. The $L 1_{0}$ martensitic structure exists up to $183 \mathrm{~K}$ and the $B 2$ austenitic structure 
starts to appear during the first order phase transition at $103 \mathrm{~K}$. Only the $B 2$ and $A 1$ phases were observed from $284 \mathrm{~K}$.

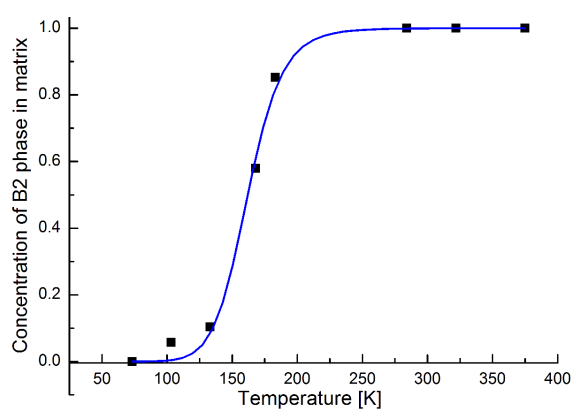

Fig. 1. Temperature evolution of $B 2$ phase content during reverse martensitic transformation in the sintered powder sample.

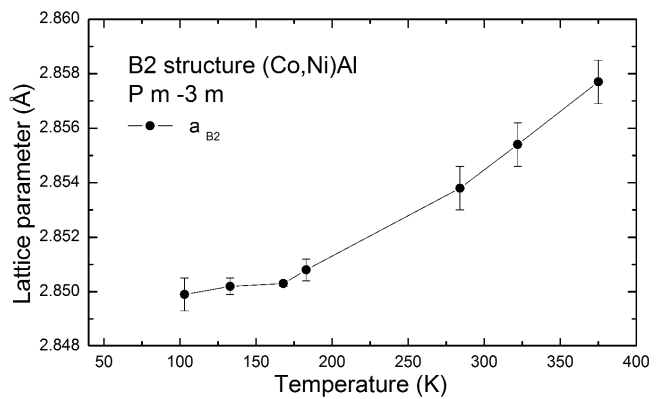

Fig. 2. The evolution of $B 2$ phase lattice parameter with temperature.

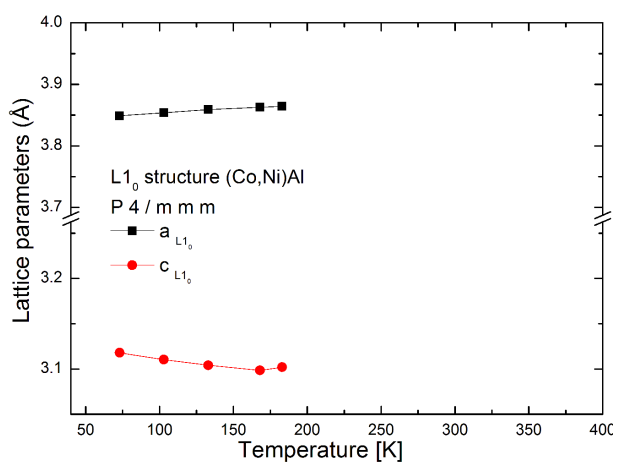

Fig. 3. The evolution of $L 1_{0}$ phase lattice parameters with temperature.

The evolution of the volume fraction of transforming phases during the reverse martensitic transformation can be seen in Fig. 1. The lattice parameters of matrix change with temperature, due to the thermal expansion, Figs. 2-4. The determined thermal expansion coefficient $-3.3 \times 10^{-5} \mathrm{~K}^{-1}$ is higher than linear thermal expansion coefficient values for cobalt $-1.3 \times 10^{-5} \mathrm{~K}^{-1}$.

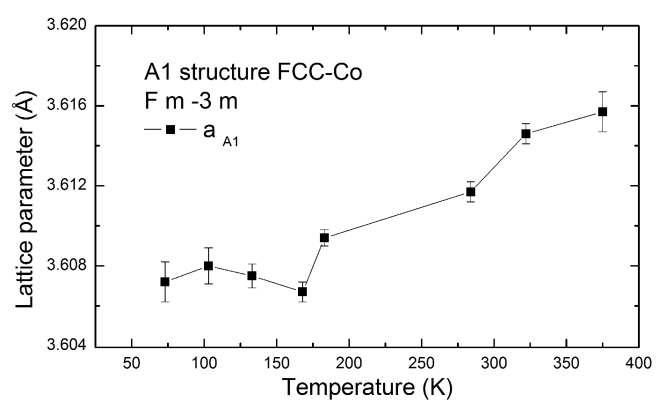

Fig. 4. The evolution of $A 1$ phase lattice parameter with temperature.

TABLE

Quantitative phase composition of the powder with respect to temperature.

\begin{tabular}{c|c|c|c}
\hline \hline$T[\mathrm{~K}]$ & $x_{A 1}[\%]$ & $x_{B 2}[\%]$ & $x_{L 1 \_0}[\%]$ \\
\hline 375 & 9 & 91 & 0 \\
322 & 13 & 87 & 0 \\
284 & 14 & 86 & 0 \\
183 & 12 & 75 & 13 \\
168 & 12 & 51 & 37 \\
133 & 13 & 9 & 78 \\
103 & 11 & 5 & 83 \\
73 & 13 & 0 & 87
\end{tabular}

The Rietveld quantitative analysis shows that the particles of fcc cobalt solid solution are present in roughly the same weight fraction (9-14 wt\%) at all temperatures, Table. There is no physical reason for the change of weight fraction of fcc cobalt solid solution particles during heating. This notion is supported by the direct comparison of the $A 1$ phase peak intensities, which are approximately constant for the whole temperature range. The observed change of the ratio between matrix and $A 1$ phase (fcc cobalt solid solution) in neutron diffraction experiment may be caused by the different diffraction power of $B 2$ and $L 1_{0}$ phases and structure effects during martensitic transformation.

\subsection{Polycrystalline bulk sample}

The bulk sample was also characterized in order to evaluate possible differences with the powder. Neutron diffraction at $89 \mathrm{~K}$ confirmed the $L 1_{0}$ and $A 1$ structures. Both $L 1_{0}$ and $B 2$ phases' diffraction peaks were observed in matrix at approximately $215 \mathrm{~K}$. The reverse martensitic transformation was almost finished at this temperature in the powder sample. Although the diffractograms are affected by the real structure and texture, we can estimate that there is a much higher amount of $L 1_{0}$ than found in powder. Only (210) and small (110) peaks of the $B 2$ phase were observed at room temperature, which is due to large size transformed $B 2$ grains, as confirmed by metallographic studies, Fig. 5 .

The reverse martensitic transformation leads to significant coarsening of the microstructure and diffrac- 


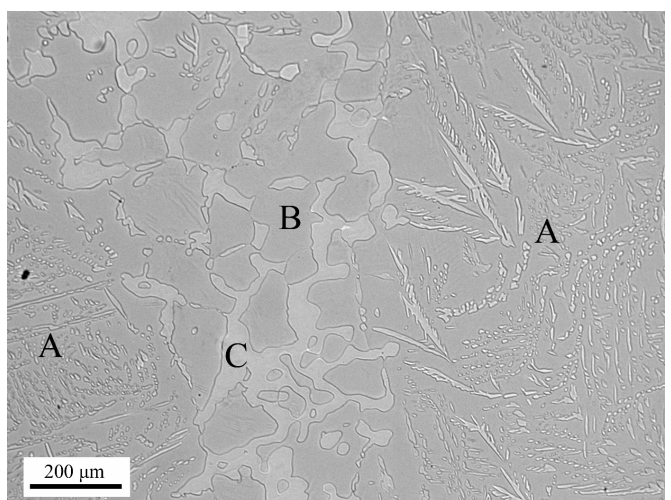

Fig. 5. The microstructure of a polycrystalline sample. Particles of fcc cobalt solid solution with two different morphologies are distributed within the $B 2$ ordered matrix. Indicated: big $B 2$ grains with $A 1$ precipitates $-\mathrm{A}$; smaller $B 2$ grain $-\mathrm{B}$; big $A 1$ particle - C.

tograms become insufficient for reliable quantitative analysis. Subsequent metallurgical studies proved that the bulk sample consists of a rather limited number of grains. The microstructure of the bulk sample is complicated by the presence of large $B 2$ grains with plenty of small fcc cobalt solid solution particles and areas of smaller $B 2$ phase grains embedded within large fcc cobalt solid solution formations, Fig. 5. Such structure originates from a complicated crystallization kinetics. Thus, sufficient statistics for representative diffraction patterns could not be obtained due to the small amount of diffracting grains. Diffraction peaks of $B 2$ austenite did not appear, though the complex microstructure of martensitic lamellae exhibited good conditions for diffraction.

\section{Discussion}

The martensitic transformation depends strongly on the thermomechanical history of the sample and the local stress distribution. Neutron diffraction proved to be efficient tool to study the phase transformation within the whole volume of the material.

Annealing and subsequent quenching create a special state of matrix with a massive amount of various phase nanoprecipitates. They are thought to be responsible for a high transformation temperature [7]. The same behavior was found in the crystals grown in our laboratory in which fcc cobalt solid solution nanoprecipitates [8] or hexagonal close packed (hcp) cobalt solid solution nanoprecipitates [9] were observed. Such small objects in the matrix can affect the shape of the matrix diffraction peaks (broadening), however the quality of the data is not good enough to perform a reliable size/strain analysis. The present experiments established reverse martensitic transformation temperatures to be $A_{\mathrm{S}}>103 \mathrm{~K}$ and $A_{\mathrm{F}} \approx 215 \mathrm{~K}$. These results agree with the values obtained on the same alloy by magnetic susceptibility measurement. In the bulk, the pinning of a significant amount of martensite was observed at $199 \mathrm{~K}$ in contrast with the transformation of the powder of the same composition, which is nearly completely transformed (approximately $98 \%$ of $B 2$ austenite) at this temperature. Unfortunately, the bulk sample diffraction profiles do not show quality for further detailed analysis. The reason of the poor quality of data is the roughening of the microstructure due to the transformation of various martensite variants into the cubic austenitic phase, as was explained in the previous section.

\section{Conclusions}

Phase transformations in the $\mathrm{Co}_{38} \mathrm{Ni}_{33} \mathrm{Al}_{29}$ alloy in powder as well as polycrystalline bulk form were studied by in situ neutron diffraction during heating. The reverse martensitic transformation in powder was observed in the temperature range $A_{\mathrm{S}}>103 \mathrm{~K}$ and $A_{\mathrm{F}} \approx 215 \mathrm{~K}$. The bulk sample transforms to martensite as well but at a higher temperature and we cannot exclude the existence of martensite pinned on precipitates at the highest temperature measured. However, since the bulk sample contains large grains, detailed interpretation of the neutron diffraction data is not reliable.

\section{Acknowledgments}

Authors would like to acknowledge the financial support from the Czech Science Foundation projects 101/ 09/0702 and P107/10/0824, the Grant Agency of the AS CR project IAA100100920 and the internal project of the Czech Geological Survey, No. 332300.

\section{References}

[1] O. Heczko, N. Scheerbaum, O. Gutfleisch, in: Nanoscale Magnetic Materials and Applications, Eds. J.P. Liu, E. Fullerton, O. Gutfleisch, D.J. Sellmyer, Springer Science+Business Media, LLC, New York 2009, p. 14-1.

[2] O. Heczko, A. Sozinov, K. Ullakko, IEEE Trans. Magn. 36, 3266 (2000).

[3] M. Hubert-Protopescu, H. Hubert, in: Ternary Alloys: A Comprehensive Compendium of Evaluated Constitutional Data and Phase Diagram. Vol. 4: Al$\mathrm{Cd}-\mathrm{Ce}$ to $\mathrm{Al}-\mathrm{Cu}-\mathrm{Ru}$, Eds. G. Petzow, G. Effenberg, VCH, Weinheim 1991, p. 234.

[4] Y. Murakami, D. Shindo, K. Oikawa, R. Kainuma, K. Ishida, Acta Mater. 50, 2173 (2002).

[5] J. Kopeček, K. Jurek, M. Jarošová, J. Drahokoupil, S. Sedláková-Ignácová, P. Šittner, V. Novák, IOP Conf. Sci.: Mater. Sci. Eng. 7, 012013 (2010).

[6] J. Kopeček, S. Sedláková-Ignácová, K. Jurek, M. Jarošová, J. Drahokoupil, P. Šittner, V. Novák, in: 8th Europ. Symp. on Martensitic Transformations, ESOMAT 2009, Eds. P. Šittner, V. Paidar, L. Heller, H. Seiner, EDP Sciences, Les Ulis Cedex 2009, p. 02013.

[7] Yu.I. Chumlyakov, I.V. Kireeva, E.Yu. Panchenko, E.E. Timofeeva, Z.V. Pobedennaya, S.V. Chusov, I. Karaman, H. Maier, E. Cesari, V.A. Kirillov, Russ. Phys. J. 51, 1016 (2008).

[8] B. Bártová, D. Schryvers, Z.Q. Yang, S. Ignácová, P. Sittner, Scr. Mater. 57, 37 (2007).

[9] B. Bártová, N. Wiese, D. Schryvers, N.J. Chapman, S. Ignácová, Acta Mater. 56, 4470 (2008). 